\title{
Study on Vibration Behavior of Doubly Clamped Silicon Nanowires by Molecular Dynamics
}

\author{
H. Yu, W. W. Zhang, S. Y. Lei, L. B. Lu, C. Sun, and Q. A. Huang \\ Key Lab of MEMS of Ministry of Education, Southeast University, Nanjing 210096, China \\ Correspondence should be addressed to H. Yu, h_yu@seu.edu.cn
}

Received 25 February 2012; Revised 1 April 2012; Accepted 19 April 2012

Academic Editor: Sergio J. Mejía-Rosales

Copyright () 2012 H. Yu et al. This is an open access article distributed under the Creative Commons Attribution License, which permits unrestricted use, distribution, and reproduction in any medium, provided the original work is properly cited.

The vibration behavior of doubly clamped silicon nanowires with square cross sections is studied by molecular dynamics method. Silicon nanowires have lengths ranging from 4.888 to $12.491 \mathrm{~nm}$ and cross sections ranging from $1.22 \mathrm{~nm} \times 1.22 \mathrm{~nm}$ to $3.39 \mathrm{~nm} \times$ $3.39 \mathrm{~nm}$. The size dependence of the resonant frequency is studied in detail. The results show that the vibration behavior of $\mathrm{Si}$ nanowire is quite different from the macroscopic beam, and the resonant frequency is much higher than the result based on the continuum theory, but close to the theoretical result based on the semicontinuum approach. Surface reconstruction can strongly affect on vibration behavior. These results demonstrate that the classic theory may not be suitable for analysis of performances of nanostructures, and the conclusion of the study has a certain practical significance on related fields.

\section{Introduction}

In last decade, resonant nanoelectromechanical systems (NEMSs) have been found in a variety of important applications, including ultrasensitive mass and force sensing [ 1 , $2]$, ultralow-power radio frequency $(\mathrm{RF})$ signal generation, timing [3-5], and switch [6]. Silicon-based nanowires and nanobeams with very small dimensions and highperformance form the basis of most experimental demonstrations in these applications, where the nanowires or the nanobeams appear either in cantilever or in doubly clamped beam resonators. Nanoscale cantilevers are preferred to be resonators in mass sensing and ultrahigh frequency applications. For example, the nanothick resonant cantilever is used to detect ppm level trace trimethylamine vapor via specific molecular adsorption by measuring the resonant frequency shift of the cantilever [7]. On the other hand, doubly clamped silicon nanowire or nanobeam is another type of resonators in nanomechanical systems. They are also very attractive to researchers. As early as the year 2007, the fundamental resonance of $215 \mathrm{MHz}$ of the doubly clamped silicon nanowire was demonstrated by Feng et al. [3]. The demonstration of mass spectrometry based on single biological molecule detection by using a doubly clamped silicon resonant nanobeam was reported in 2009 [8]. Therefore, the resonant or vibration behavior of the silicon nanowire is very important to the mass sensing and other applications and has been widely studied by researchers.

In the aspect of theoretical study, some of researches applied equations and software based on continuum assumption to nanowires when they studied the resonance of silicon nanowires and the relationship between resonant frequency and Young's modulus $[3,9,10]$. However, because of the low dimension of the nanowire, the mechanical properties and thus the vibration behavior may deviate from their microscopic and macroscopic counterparts. It means that classical theories based on continuum assumptions or computational design tools, which have been developed for microstructures and macrostructures, may not be directly applicable for the nanostructures. Some approaches have been proposed to extend classical continuum theories to nanostructured materials. By considering the discrete nature of nanomaterial, Sun and Zhang developed a semicontinuum model to calculate Young's modulus of the ultrathin film with a simple cubic lattice [11]. This approach was then developed to study Young's modulus of silicon nanoplate [12]. Based on this approach, a multiscale model was built to analyze the deflection and resonant frequency of silicon nanobeam $[13,14]$. On the other hand, molecular dynamics method 


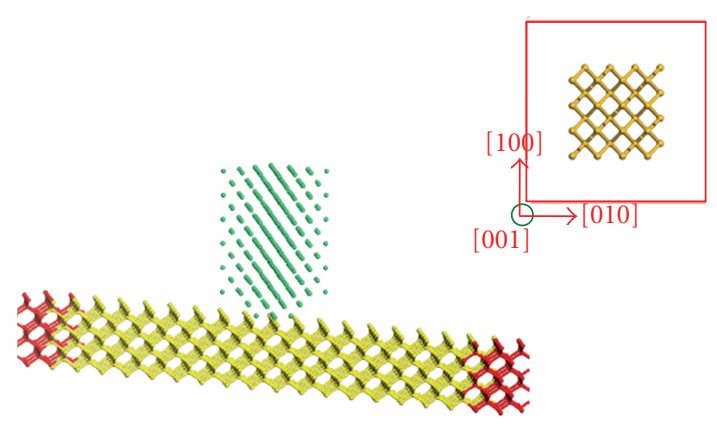

(a)

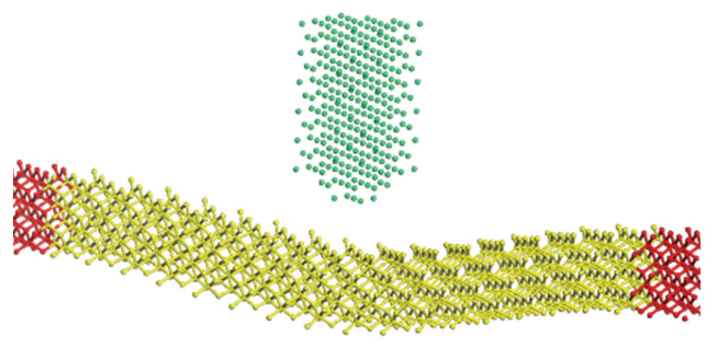

(b)

FIgURE 1: Model of a doubly clamped silicon nanowire and one AFM probe: (a) before optimization and (b) after optimization.

is often used to study and simulate the mechanical and resonant properties of nanowires. Park et al. and Kim et al. $[15,16]$ simulated the flexural and longitudinal vibrations of silicon nanocantilevers by molecular dynamics method. They deduced the elastic modulus and Young's modulus for nanothick silicon cantilevers from the natural frequency equation based on the continuum theory.

In this paper, we study the resonant behavior of doubly clamped silicon nanowires by using a specific computer code, Forcite, which is based on molecular dynamics method. Our results are in well agreement with the results based on the multiscale model but much higher than those based on continuum theory.

\section{Construction of Simulation Model}

Simulation model of one doubly clamped silicon nanowire with a square cross section is built as shown in Figure 1(a) (yellow atoms). The red atoms at two ends of the silicon nanowire are clamped. The cross section and the orientation of the silicon nanowire are depicted in Figure 1, too. Periodic boundary condition is used in the longitudinal direction of Si nanowire, and lateral surfaces are under free boundary conditions in vacuum. The simulation has been carried out at the average temperature of $298 \mathrm{~K}$ with a time step of $1 \mathrm{fs}$. Compass (Condensed-phase Optimized Molecular Potential for Atomistic Simulation Studies) force-field is selected when simulation is performed.

To actuate the nanowire, an AFM probe is constructed above the nanowire, which is denoted by green atoms in Figure 1. After structure optimization, the nanowire begins to deflection as shown in Figure 1(b), because Van der Waals force exists between the AFM and the nanowire. Then the AFM is removed and the simulation starts. Because of the initial deflection, the nanowire will vibrate freely.

\section{Simulation Results and Discussion}

Figure 2 shows the kinetic and potential energy of the silicon nanowire when the nanowire is vibrating. It can be seen that after undergoing very short transient oscillations, the kinetic energy and potential energy start oscillating almost in one uniform period. At the maximum point of the kinetic

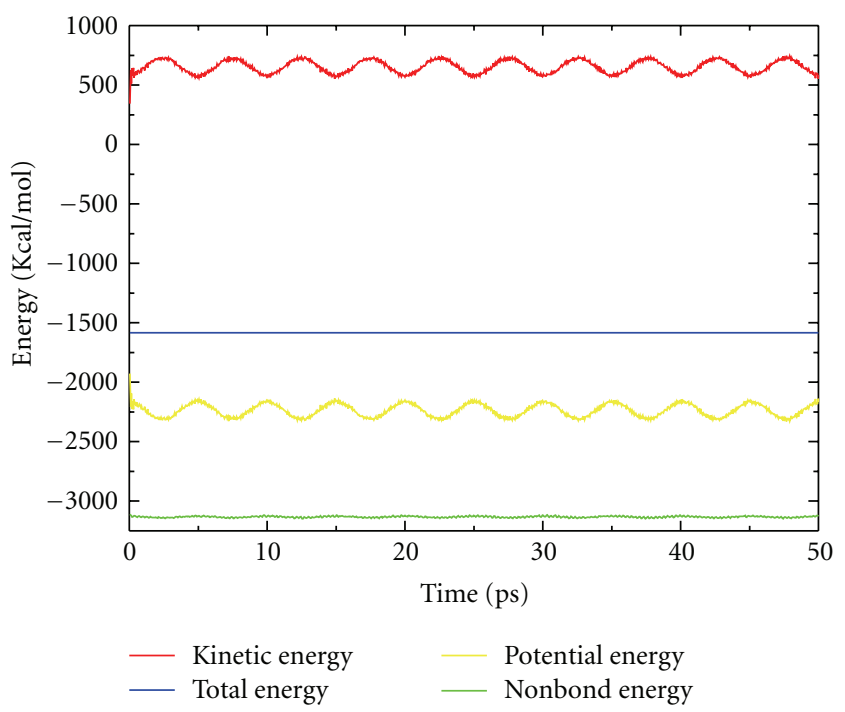

FIGURE 2: Energy of the nanowire while vibrating.

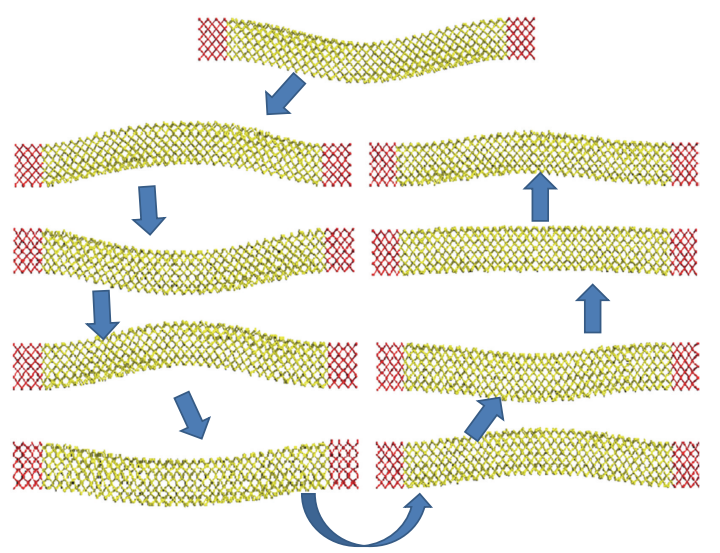

FIGURE 3: Vibration traces of the silicon nanowire in one oscillation period.

energy, the potential energy reaches to the minimum, and vice versa. Exchange between the kinetic energy and the potential energy occurs during one period.

Figure 3 shows the vibration traces of the nanowire in the successive time moment during one oscillation period. It is 


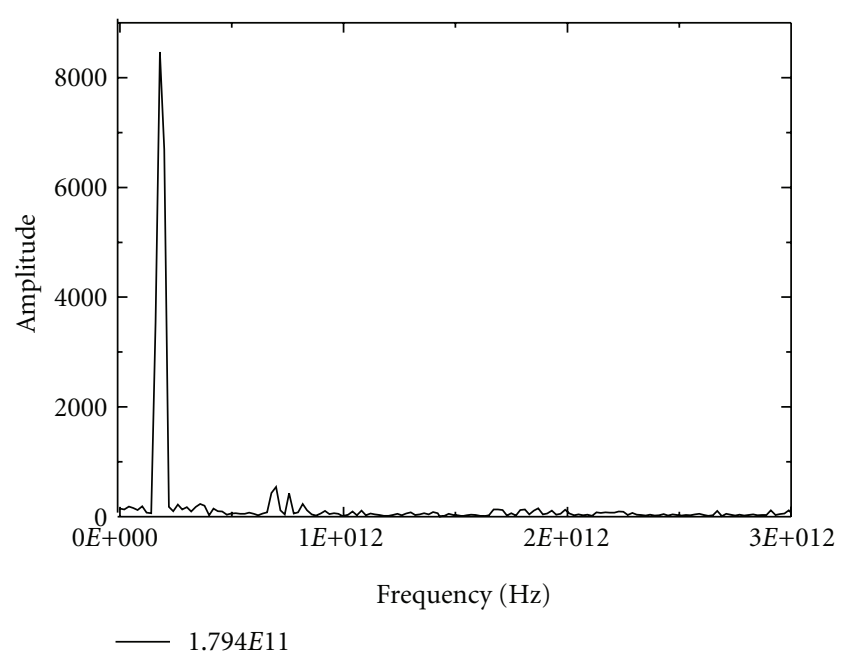

FIgURE 4: Frequency response of a silicon nanowire.

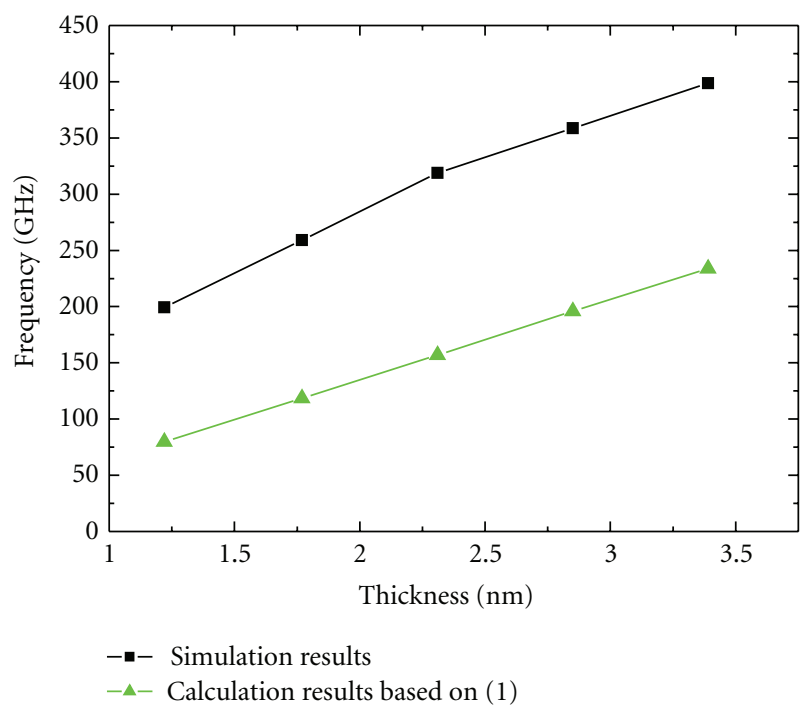

FIGURE 5: Fundamental frequency as a function of nanowire thickness: simulation results by MD software (black), and calculation results based on (1) (green) while Young's modulus coming from $[15,16]$. All nanowires keep the same length of $9.232 \mathrm{~nm}$ and the same width of $2.85 \mathrm{~nm}$.

seen that the vibration mode is the first mode and the motion of the nanowire in one oscillation period is different from that of a macroscopic beam.

The frequency response of the silicon nanowire can be obtained by performing FFT of the kinetic energy or the potential energy. Figure 4 shows the frequency response of the nanowire with a length of $9.232 \mathrm{~nm}$. The cross section is $1.22 \mathrm{~nm} \times 1.22 \mathrm{~nm}$. It can be seen that the fundamental frequency is about $179.4 \mathrm{GHz}$.

The vibrations of nanowires with different thicknesses are studied. The fundamental frequency as a function of thickness of the nanowire is shown in Figure 5 (black).
As we have known, the first mode frequency $\omega_{0}$ and the $j_{\text {th }}$ mode frequency $\omega_{j}$ for a doubly clamped Euler-Bernoulli beam can be written as [17]

$$
\begin{gathered}
\omega_{0}=\sqrt{\frac{E I}{m l^{4}}}, \\
\omega_{j}=(j \pi)^{2} \omega_{0} \quad(j=1,2, \ldots),
\end{gathered}
$$

where $E$ is Young's modulus, $m$ is mass per unit length, $m=\rho A, \rho$ is the density of the beam, $A$ is the area of the cross section, and $l$ and $I$ are the beam length and the mass moment of inertia, respectively. Now we apply this model to calculate fundamental frequencies of doubly clamped silicon nanowires. Young's modulus in (1) takes the value obtained by Park et al. $[15,16]$ for silicon nanowire. The results calculated from (1) for nanowires with different thicknesses are also shown in Figure 5 (green).

It is noticed that there is a big deviation between simulation results and calculation results based on (1). The deviation is larger than $60 \%$. This means that the classic theory may not be suitable for analysis of performance of nanostructures, since (1) is built based on continuum assumption, and the discrete nature must be considered in the model for nanostructures. By considering the discrete nature in thickness direction of nanobeam, a multiscale model is proposed to study the vibration frequency of silicon nanobeam [14]. In this model, the fundamental frequency of the doubly clamped silicon nanobeam is described as

$$
\begin{aligned}
& f_{0}=\frac{\omega_{0}}{2 \pi} \\
& = \begin{cases}\frac{\pi}{3 l^{2} t} \sqrt{\frac{2 k a}{\rho} \times \frac{(N+1)(2 N+1)}{2+t^{2}},} & N \leq 3, \\
\frac{2 \pi}{l^{2}} \sqrt[\begin{array}{c}
\frac{k a}{3 \rho N}\left[\frac{1}{18}(N-3)(N-2)(2 N-5)\right. \\
\left.+\frac{3(N-3)^{2}+12(N-3) t+14 t^{2}}{t(2+t)^{2}}\right],
\end{array}]{ } & N>3,\end{cases}
\end{aligned}
$$

where $l, \rho$, and $a$ are the length, the density, and the lattice constant of the silicon nanobeam, respectively. $N$ is the number of lattices in thickness, $k$ is the spring constant between two silicon atoms, $2.614 \times 10^{2} \mathrm{~N} / \mathrm{m}$, and $t$ is the relaxation constant. For nonsurface relaxation, $t=1$. Based on this multiscale approach, resonant frequencies for silicon nanowires are calculated and compared with our simulation results, as shown in Figure 6. It is clear that the discrepancy between them is smaller than the deviation in Figure 5. The deviation here is smaller than $20 \%$. This figure shows that our results are in well agreement with the multiscale model results.

When the length of the nanowire is changed, the vibration behavior will change, too. The fundamental frequencies of doubly clamped silicon nanowires with different lengths are studied. Figure 7 shows the resonant frequency as a function of length of the silicon nanowire. The fundamental 


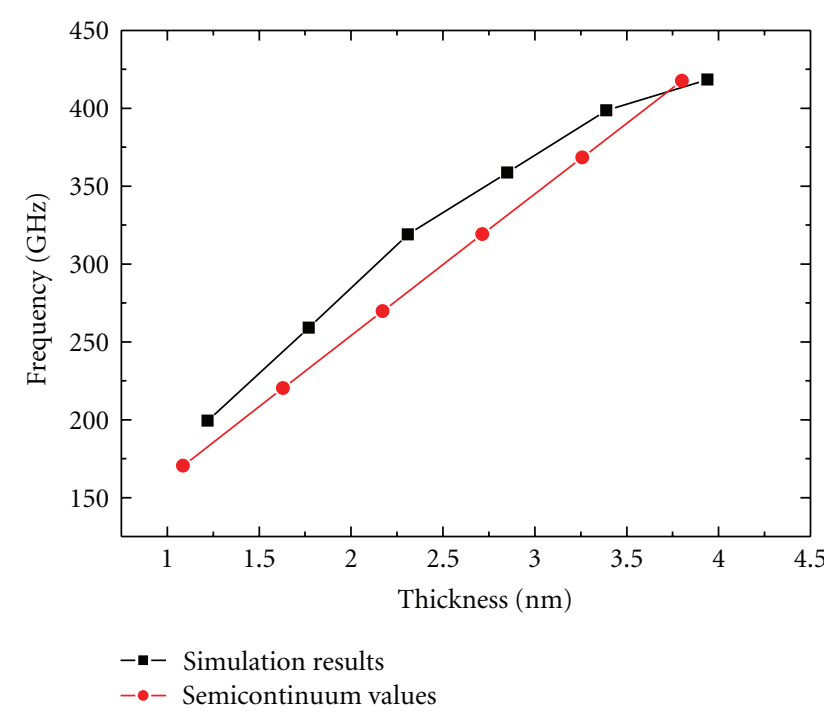

FIGURE 6: Fundamental frequency as a function of nanowire thickness: simulation results by MD software (black), and calculation results based on multiscale model (red). The length of nanowire is $9.232 \mathrm{~nm}$.

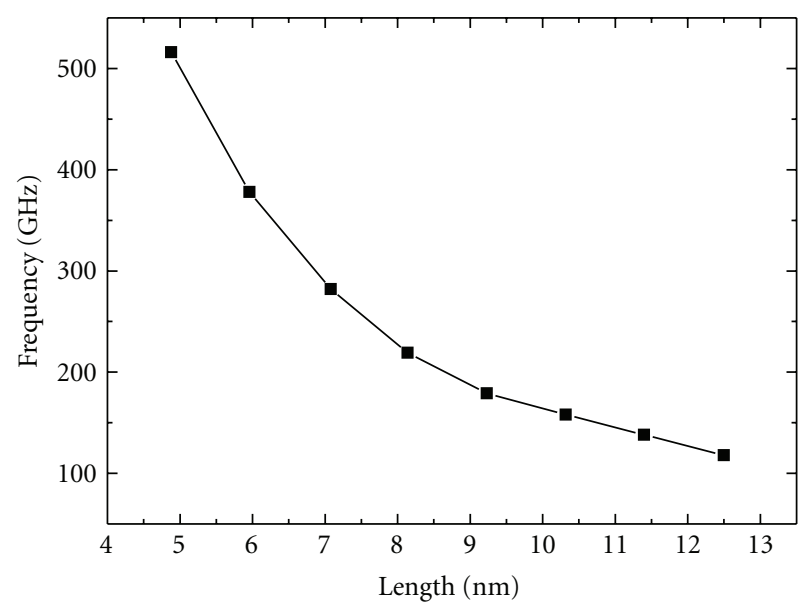

FIGURE 7: Fundamental frequency as a function of length of silicon nanowire. The width and thickness of nanowires are both of $1.22 \mathrm{~nm}$.

frequency decreases monotonically with the length increasing.

When the width is changed, the frequency response of the nanowire does not change significantly, as shown in Figure 8. This concludes that the transverse vibration frequency is not related to the width of nanowire too much, which is similar with the result based on continuum theory.

Surface reconstruction can strongly impact on the elasticity of silicon nanoplate [18-20]. Therefore, the effect of surface reconstruction on vibration frequency of silicon nanowire is also studied in detail. The $(2 \times 1)$ dimmer reconstruction is considered on four of lateral surfaces of the silicon nanowire. Figure 9 shows the vibration frequencies with and without reconstruction for nanowires with different cross sections. It can be seen that the frequency under surface

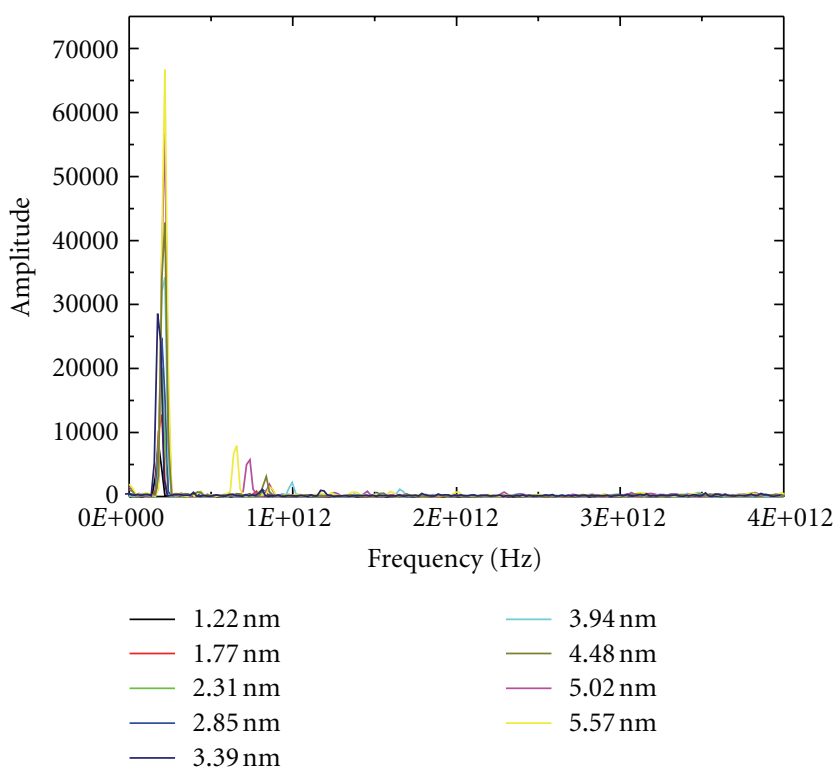

FIGURE 8: Frequency responses of doubly clamped nanowires with different widths.

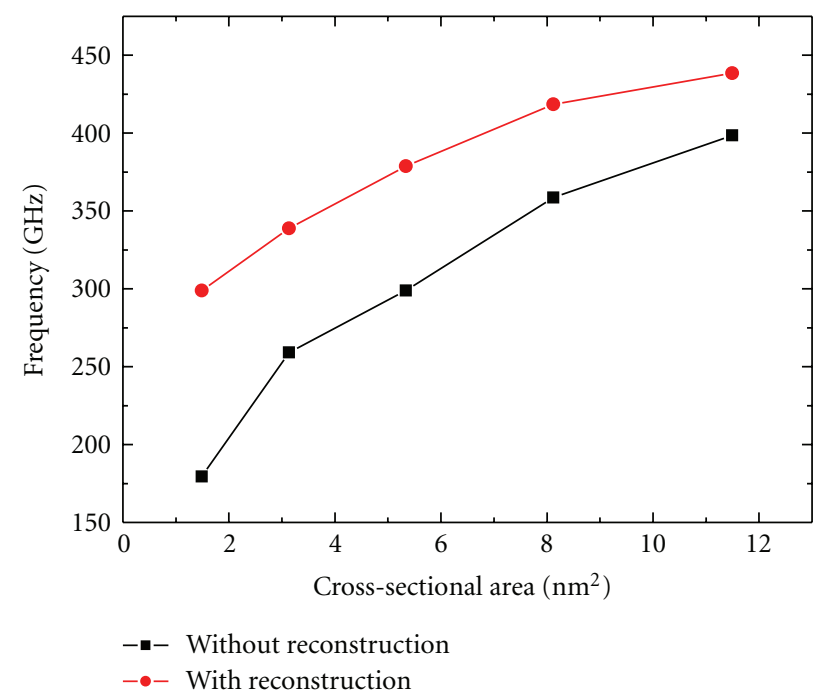

Figure 9: Resonant frequency for nanowires with different cross sections with and without reconstructions on lateral surfaces.

reconstruction is much higher than that without reconstruction. The frequency is almost doubled by surface reconstruction for the nanowire with the cross section of $1.488 \mathrm{~nm}^{2}$. Because of the surface reconstruction, the nanowire becomes stiffer, and Young's modulus becomes larger, resulting in the resonant frequency increasing significantly. The size of cross section is smaller, the change of resonant frequency is greater, since the surface-to-volume ratio is higher for the nanowire with a smaller cross section.

\section{Conclusions}

Vibration behavior of doubly clamped silicon nanowires is studied by molecular dynamics software. The results are 
different from those based on classic continuum theory but are close to the results obtained from semicontinuum approach. Resonant frequency of doubly clamped silicon nanowires strongly depends on the length and thickness of the nanowire. Surface reconstruction has strong impact on the vibration behavior of the silicon nanowire, especially on the nanowire with smaller size. The results show that the classic theory may not be suitable for analysis of the vibration of silicon nanowires.

\section{Acknowledgment}

This project is supported by the National Science Foundation of China (Grant no. 61001044).

\section{References}

[1] Y. T. Yang, C. Callegari, X. L. Feng, K. L. Ekinci, and M. L. Roukes, "Zeptogram-scale nanomechanical mass sensing," Nano Letters, vol. 6, no. 4, pp. 583-586, 2006.

[2] K. L. Ekinci and M. L. Roukes, "Nanoelectromechanical systems," Review of Scientific Instruments, vol. 76, no. 6, Article ID 061101, 12 pages, 2005.

[3] X. L. Feng, R. R. He, P. D. Yang, and M. L. Roukes, "Very high frequency silicon nanowire electromechanical resonators," Nano Letters, vol. 7, no. 7, pp. 1953-1959, 2007.

[4] X. L. Feng, C. J. White, A. Hajimiri, and M. L. Roukes, "A self-sustaining ultrahigh-frequency nanoelectromechanical oscillator," Nature Nanotechnology, vol. 3, no. 6, pp. 342-346, 2008.

[5] N. Liu, F. Giesen, M. Belov et al., "Time-domain control of ultrahigh-frequency nanomechanical systems," Nature Nanotechnology, vol. 3, no. 12, pp. 715-719, 2008.

[6] E. F. Arkan, D. Sacchetto, I. Yildiz, Y. Leblebici, and B. E. Alaca, "Monolithic integration of Si nanowires with metallic electrodes: NEMS resonator and switch applications," Journal of Micromechanics and Microengineering, vol. 21, Article ID 125018, 9 pages, 2011.

[7] Y. L. Yang, X. Y. Xia, X. H. Gan, P. C. Xu, H. T. Yu, and X. X. Li, "Nano-thick resonant cantilevers with a novel specific reaction-induced frequency-increase effect for ultrasensitive chemical detection," Journal of Micromechanics and Microengineering, vol. 20, no. 5, Article ID 055022, 5 pages, 2010.

[8] A. K. Naik, M. S. Hanay, W. K. Hiebert, X. L. Feng, and M. L. Roukes, "Towards single-molecule nanomechanical mass spectrometry," Nature Nanotechnology, vol. 4, no. 7, pp. 445450, 2009.

[9] M. Belov, N. J. Quitoriano, S. Sharma, W. K. Hiebert, T. I. Kamins, and S. Evoy, "Mechanical resonance of clamped silicon nanowires measured by optical interferometry," Journal of Applied Physics, vol. 103, no. 7, Article ID 074304, 7 pages, 2008.

[10] Y. K. Feng, Y. L. Liu, and B. Wang, "Finite element analysis of resonant properties of silicon nanowires with consideration of surface effects," Acta Mechanica, vol. 217, no. 1-2, pp. 149-155, 2011.

[11] C. T. Sun and H. T. Zhang, "Size-dependent elastic moduli of platelike nanomaterials," Journal of Applied Physics, vol. 93, no. 2, pp. 1212-1218, 2003.

[12] J. Wang, Q. A. Huang, and H. Yu, "Size and temperature dependence of Young's modulus of a silicon nano-plate,"
Journal of Physics D, vol. 41, no. 16, Article ID 165406, 5 pages, 2008.

[13] F. Bao, H. Yu, Q. R. Lu, and Q. A. Huang, "Multi-scale model for load-deflection of Si nanobeams," Chinese Journal of Semiconductors, vol. 28, no. 12, pp. 1979-1982, 2007.

[14] Q. R. Lu, F. Bao, H. Yu, and Q. A. Huang, "A semicontinuum model of nature frequency for nanoscaled clamped silicon beam," Chinese Journal of Sensors and Actuators, vol. 21, no. 3, pp. 469-472, 2008.

[15] S. H. Park, J. S. Kim, J. H. Park, J. S. Lee, Y. K. Choi, and O. M. Kwon, "Molecular dynamics study on size-dependent elastic properties of silicon nanocantilevers," Thin Solid Films, vol. 492, no. 1-2, pp. 285-289, 2005.

[16] J. S. Kim, S. H. Park, J. H. Park, and J. S. Lee, "Molecular dynamics simulation of elastic properties of silicon nanocantilevers," Nanoscale and Microscale Thermophysical Engineering, vol. 10, no. 1, pp. 55-65, 2006.

[17] W. J. Bottega, Engineering Vibrations, CRC Taylor \& Francis, 2006.

[18] H. W. Shim, L. G. Zhou, H. C. Huang, and T. S. Cale, "Nanoplate elasticity under surface reconstruction," Applied Physics Letters, vol. 86, no. 15, Article ID 151912, 3 pages, 2005.

[19] L. G. Zhou and H. C. Huang, "Are surfaces elastically softer or stiffer?” Applied Physics Letters, vol. 84, no. 11, pp. 1940-1942, 2004.

[20] W. W. Zhang, Q. A. Huang, H. Yu, and L. B. Lu, "Sizedependent elasticity of silicon nanowires," Advanced Materials Research, vol. 60-61, pp. 315-319, 2009. 

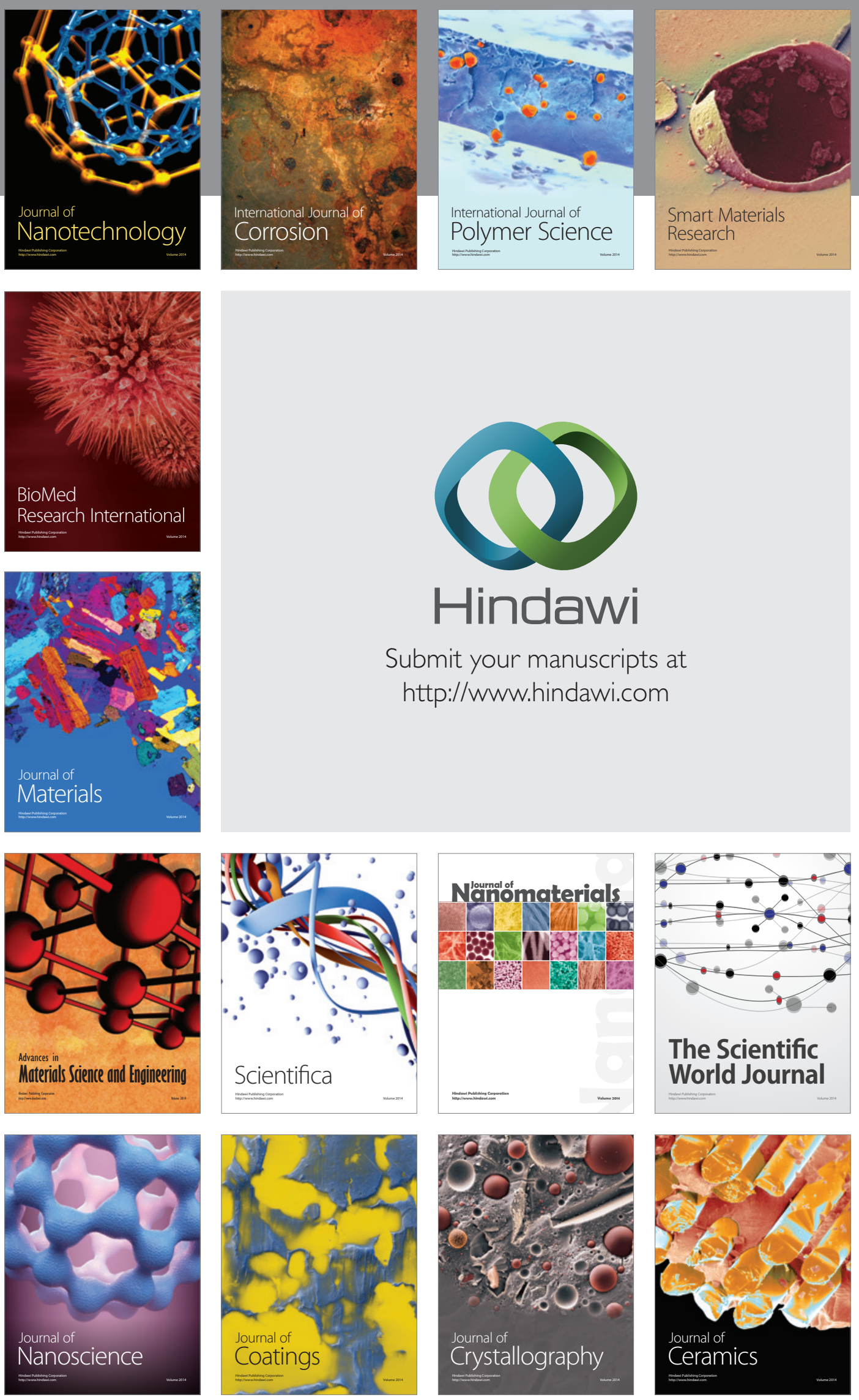

The Scientific World Journal

Submit your manuscripts at

http://www.hindawi.com

\section{World Journal}

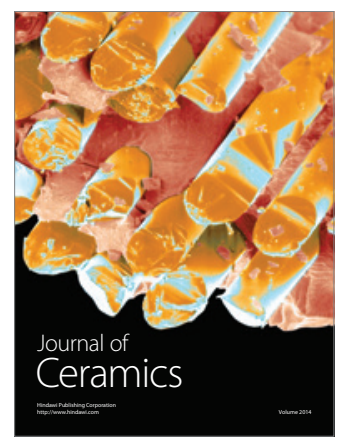

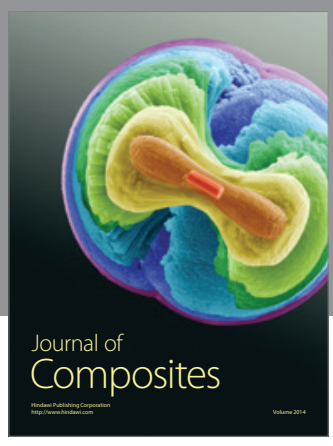
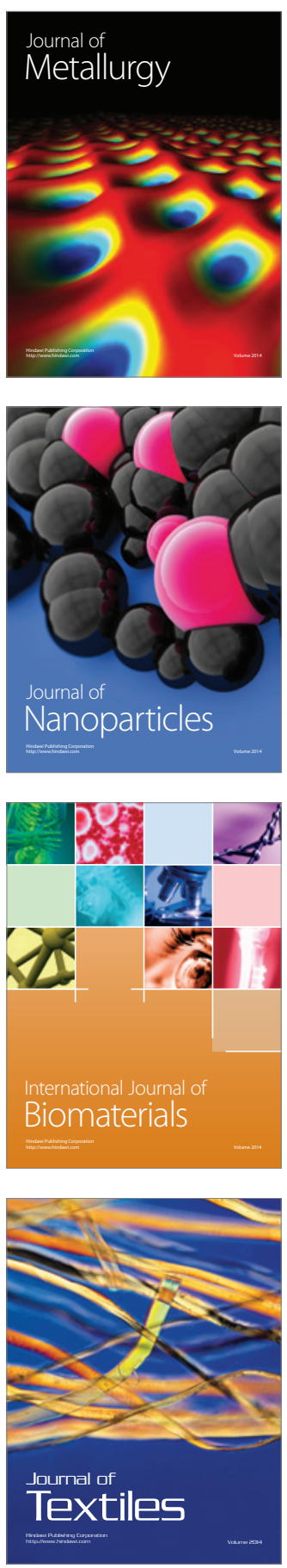